\title{
Geometric Data Manipulation with Clifford Algebras and Möbius Transforms
}

\author{
Mijail Guillemard*, Armin Iske and Udo Zölzer
}

\begin{abstract}
We propose the usage of Möbius transformations, defined in the context of Clifford algebras, for geometrically manipulating a point cloud data lying in a vector space of arbitrary dimension. We present this method as an application to signal classification in a dimensionality reduction framework. We first discuss a general situation where data analysis problems arise in signal processing. In this context, we introduce the construction of special Möbius transformations on vector spaces $\mathbb{R}^{n}$, customized for a classification setting. A computational experiment is presented indicating the potential and shortcomings of this framework.
\end{abstract}

Keywords. Data classification, signal processing, Clifford algebras, Möbius transforms, dimensionality reduction, short term Fourier analysis.

\section{Introduction and General Framework}

In the field of signal processing, a fundamental strategy is the usage of Fourier transforms or wavelet analysis for detecting and filtering particular components of a signal. These concepts provide a powerful framework used in multiple theoretical and application fields. But the ever increasing complexity of signal data requires more sophisticated analysis tools. In the last decade, significant progress has been made in the field of data analysis and dimensionality reduction inspired by geometrical and topological concepts, see [5]. Some algorithms based on concepts from differential geometry are Whitney embedding based methods, isomap, LTSA, Laplacian eigenmaps, Riemannian normal coordinates, to mention but a few. In parallel developments, probabilistic conditions and numerical algorithms (e.g. persistent homology) have provided new tools for reconstructing the homology of a submanifold $\mathcal{M} \subset \mathbb{R}^{n}$ from a finite dataset $X=\left\{x_{i}\right\}_{i=1}^{m} \subset \mathcal{M}$. Inspired by these developments, we propose a framework based on Clifford geometry for signal classification, as an addition to the manifold learning and dimensionality reduction toolbox.

${ }^{*}$ Corresponding author 
For describing the basic ingredients of our framework, we consider datasets $X_{g}$ and $X_{h}$ as subsets of $\mathbb{R}^{n}$. Modern clustering techniques (support vector machines, kernel methods, spectral clustering, etc.) can identify clusters $X_{g}$ and $X_{h}$ by constructing features that geometrically separate these sets. The difficulty lies in the complex nonlinear geometrical properties of the datasets, as they might present challenging scenarios for clustering methods. Classification methods can be seen as a further step, where, given an unknown point $x \in \mathbb{R}^{n}$, we want to decide to which cluster it belongs. Our proposal is to use the powerful concept of geometric Clifford algebras to manipulate the geometry of $X_{g}$ and $X_{h}$ in order to simplify their geometric properties and to improve classification methods. As a step towards this goal, we propose the usage of Möbius transforms in $\mathbb{R}^{n}$ as defined in [1]. In our contribution, we show how to use nonlinear (hyperbolic) Möbius transforms to design maps that "shrink" a particular region centered around an (attractor) point in $\mathbb{R}^{n}$. The resulting transform would then, under the right conditions, simplify the classification task of deciding whether or not a point belongs to a given cluster.

To explain the application of this framework to signal processing, we consider datasets $X_{f}=X_{g} \cup X_{h}=\left\{x_{i}\right\}_{i} \subset \mathbb{R}^{n}$, constructed from a signal $f=g+h$ by taking consecutive (windowed) chunks $x_{i}$ of $f$ (as classically performed in short term Fourier analysis or wavelet theory). We use a transform $T$ (e.g. power spectrum or wavelet transforms) applied to each element $x_{i}$, and obtain $T\left(X_{f}\right):=\left\{T\left(x_{i}\right)\right\}_{i}$. The general objective is to explore the geometry and topology of $T\left(X_{f}\right)$ to obtain information about $f, g$ and $h$.

An important tool in the analysis of $T\left(X_{f}\right)$ is a dimensionality reduction map $R$ that meaningfully projects $T\left(X_{f}\right)$ into a lower dimensional Euclidean space $\mathbb{R}^{d}, d<n$. This dimensionality reduction step is expected to improve computational tasks for the analysis of $T\left(X_{f}\right)$. In a typical scenario, $X_{f}$ is embedded in a high-dimensional space $\mathbb{R}^{n}$, although its intrinsic dimension might be small An important assumption is to consider $T\left(X_{f}\right)$ as a sampling of $\mathcal{M}, T\left(X_{f}\right) \subset \mathcal{M}$, where $\mathcal{M}$ is a submanifold of $\mathbb{R}^{n}$. More generally, $\mathcal{M}$ can be seen as a topological space constructed as the geometric realization in $\mathbb{R}^{n}$ of a simplicial complex or a $\mathrm{CW}$-complex. In this setting, a suitable dimensionality reduction map $R: \mathcal{M} \subset \mathbb{R}^{n} \rightarrow \mathcal{M}_{R} \subset \mathbb{R}^{d}$ outputs by $\mathcal{M}_{R}$ a low-dimensional diffeomorphic (or homeomorphic) copy of $\mathcal{M}$.

In our contribution, the set $R\left(T\left(X_{f}\right)\right)$ is further manipulated with a Möbius map $\mathfrak{f}: \mathbb{R}^{d} \rightarrow \mathbb{R}^{d}$ that modifies the geometry of the clusters $R\left(T\left(X_{h}\right)\right.$ ) and $R\left(T\left(X_{g}\right)\right)$ ). The objective is to use the modified geometrical properties of the resulting sets $\mathfrak{f}\left(R\left(T\left(X_{g}\right)\right)\right)$ and $\mathfrak{f}\left(R\left(T\left(X_{h}\right)\right)\right)$, as these can be useful in the subsequent classification algorithms. This is in analogy to the treatment of nonlinear clustering problems, e.g., by using kernel methods and support vector machines. However, the novelty in our ideas is the usage of Clifford algebras for manipulating point clouds and construct meaningful Möbius transforms in higher dimensions. 


\section{Dimensionality Reduction}

A standard way for representing experimental information is given by the concept of point cloud data (PCD), defined as a finite set of vectors $X=$ $\left\{x_{k}\right\}_{k=1}^{m} \subset \mathbb{R}^{n}$. The dimensional reduction problem considers the case when much of the information described by $X$ is redundant and can be discarded by constructing a low dimensional representation $Y=\left\{y_{k}\right\}_{k=1}^{m} \subset \mathbb{R}^{d}$, with $d \ll n$. The main objective is to construct a dataset $Y$ such that certain characteristics of $X$ are conserved. For instance, the objective in Multidimensional Scaling (MDS) is to find $Y$ satisfying $\left\|y_{i}-y_{j}\right\| \approx\left\|x_{i}-x_{j}\right\|$ for all $i, j \in\{1, \ldots, m\}$. Another example is the case when the dataset $X$ lies in the vicinity of an hyperplane in $\mathbb{R}^{n}$ : for this situation the goal of Principal Component Analysis (PCA) is to construct $Y$ by projecting the set $X$ in this hyperplane. The low-dimensional dataset $Y$ can then be used for analysis or classification purposes.

In manifold learning, it is assumed that the elements $x_{i}$ are points lying in (or close to) a manifold $\mathcal{M}$. We consider the case $X \subset \mathcal{M}$, namely, $X$ is sampled from $\mathcal{M}$, a $p$-dimensional smooth compact submanifold of $\mathbb{R}^{n}$. Assuming the existence of a manifold is a reasonable hypothesis being fulfilled in relevant applications. As in dimensionality reduction, the objective is also to construct a low dimensional representation $Y=\left\{y_{1}, \ldots, y_{m}\right\} \subset \mathbb{R}^{d}, d \ll n$, that conserves some characteristics of the dataset $X$. Now, the geometrical structure introduced by $\mathcal{M}$ will play a crucial role in the algorithm design. Due to the Whitney embedding theorem (which states that any connected smooth $p$-dimensional manifold can smoothly be embedded in $\mathbb{R}^{2 p+1}$ ) we require some conditions for the dimensions in this formulation, namely, $2 p+$ $1 \leq d \leq n$. Our problem can also be formulated as the search for an adequate embedding $E$ of the $p$-dimensional submanifold $\mathcal{M} \subset \mathbb{R}^{n}$ in $\mathbb{R}^{d}$, with $E$ : $\mathcal{M} \subset \mathbb{R}^{n} \rightarrow \Omega \subset \mathbb{R}^{d}, X \subset \mathcal{M}, Y \subset \Omega, \Omega$ a $p$-dimensional submanifold, and $2 p+1 \leq d \leq n$.

For some applications, assuming the existence of a manifold $\mathcal{M}$ might be too restrictive. In this case the strategy would be to construct a finite metric space from $X$, and analyze its properties using topological constructions, as Čech complexes, Vietoris-Rips complexes, etc (see [2]). An important additional topic in this field are density conditions on the finite dataset $X$ (with respect to $\mathcal{M}$ ) in order to guarantee a meaningful usage of $\mathcal{M}$ and its structure. For the case of manifolds, recent results ensuring the correct computation of the homology of $\mathcal{M}$ using $X$ have been presented in [6]. For more general topological spaces, the framework of persistent homology offers robust tools for computing homological information of $\mathcal{M}$ using as input the finite samples $\left\{x_{k}\right\}_{k=1}^{m}[7]$.

\subsection{Nonlinear Dimensionality Reduction}

In order to handle point cloud data with a more complex geometry, a rich variety of algorithms have been proposed in the last few years. We describe 
here, as a representative example, the isomap algorithm. The isomap algorithm computes geodesic distances by considering the shortest path between groups of neighboring points. This procedure first identifies neighbor points using a $k$-nearest neighbors or $\epsilon$ radius criteria. Once the neighboring points are identified, the geodesic distance between two given points is computed by finding minimum connecting paths. As soon as the geodesic distances for the given dataset are obtained, the MDS algorithm can be applied: by solving an optimization problem, we construct a configuration of points $Y$ in a lower dimensional space that matches the distances in the original dataset $X$. The main points are summarized in the following list of steps.

1 Neighborhood graph construction: Define a graph where each vertex is a datapoint, and each edge connects two points if they fulfill an $\epsilon$-radius or $k$-nearest neighbors criterium.

2 Geodesic distance construction: Compute the geodesic distance between each pair of point using the graph by finding the shortest paths between the points.

$3 d$-dimensional embedding: Use the geodesic distance in a MDS algorithm for computing a $d$-dimensional embedding.

By using the geodesic distances we can construct a configuration of points representing a more accurate representation of the point cloud data.

\subsection{Interactions between Signal Processing and Dimensionality Reduction}

It is desirable to work with analysis techniques that combine signal processing transforms with dimensionality reduction methods. In this case, the basic objects are the manifold $\mathcal{M}$, the data samples $X=\left\{x_{i}\right\}_{i=1}^{m}$ taken from $\mathcal{M}$, and a diffeomorphism $\mathcal{A}: \Omega \rightarrow \mathcal{M}$, where $\Omega$ is the low-dimensional copy of $\mathcal{M}$ to be reconstructed via dimensionality reduction. Here, the only algorithmic input is the dataset $X$, but with the assumption that we can reconstruct topological information of $\mathcal{M}$ with $X$ (see for instance [6]). The other basic object in our scheme is a signal processing map $T: \mathcal{M} \rightarrow \mathcal{M}_{T}$, which may be based on Fourier analysis, wavelet transforms, or convolution filters, together with the resulting set $\mathcal{M}_{T}:=\{T(p), p \in \mathcal{M}\}$ of transformed data. The following diagram shows the basic situation.

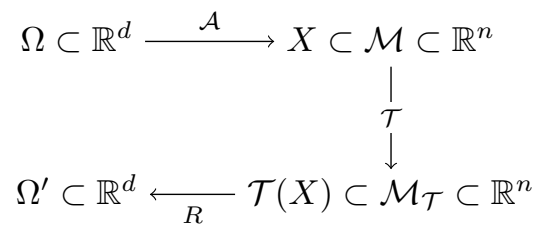

The main objective is to find an approximation of $\Omega$, denoted $\Omega^{\prime}=$ $R\left(\mathcal{M}_{T}\right)$, by using a suitable dimensionality reduction map $R$. Some properties of $\Omega$ and $\Omega^{\prime}$ may differ depending on the dimensionality reduction technique, but the target is to construct $\Omega^{\prime}$, so that geometrical and topological properties of $\Omega$ are recovered. 


\section{The Clifford Algebra Toolbox}

The basic ingredient in our framework is a mechanism for manipulating the geometry of a point cloud data. For this purpose we shortly recall the interplay between Clifford algebras, exterior algebras, and geometric algebras. These tools can be particularly important in the design of signal separation and classification algorithms, as they provide efficient algebraic methods for manipulating geometrical data. Here, we focus on the construction of a fundamental nonlinear map, the Möbius transformation in $\mathbb{R}^{n}$.

A Clifford algebra is a generalization of the complex numbers defining a product in the vector space $V=\mathbb{R}^{n}$ with similar properties as the complex multiplication. More precisely, let $q_{n}$ be the standard Euclidean inner product in $\mathbb{R}^{n}$. Then, the Clifford algebra $\mathrm{Cl}_{n}=\mathrm{Cl}\left(\mathbb{R}^{n}, q_{n}\right)$ is an associative algebra generated by the elements of $\mathbb{R}^{n}$ subject (only) to the relation $v^{2}=-q_{n}(v, v) 1, v \in \mathbb{R}^{n}$. More general bilinear forms $q_{n}$ are of relevance in many fields (e.g. differential geometry or noncommutative geometry [4]), but here we restrict ourselves to the case of the standard inner product. We remark that the notation $\mathrm{Cl}_{n}$ refers to what is know in the standard literature as $\mathrm{Cl}_{n, 0}$, where the subscript refers to the way the nondegenerate quadratic form in the definition of a Clifford algebra is written in diagonal form. Here, our notation then differs, and we use $\mathrm{Cl}_{n}$ to denote $\mathrm{Cl}_{0, n}$. An explicit construction of a Clifford algebra is given by considering $\mathrm{Cl}_{n}$ to be the associative algebra over the real numbers generated by elements $e_{1}, \ldots, e_{n}$ subject to the relations $e_{i}^{2}=-1, e_{i} e_{j}=-e_{j} e_{i}, i \neq j$ (anti-commutativity). Every element $a \in \mathrm{Cl}_{n}$ can be represented as

$$
a=\sum_{J} a_{J} e_{J}, \quad e_{J}:=e_{j_{1}} \ldots e_{j_{k}}, \quad \text { with } a_{J} \in \mathbb{R},
$$

where the sum ranges over all multi-indices $J=\left\{j_{i}\right\}_{i=1}^{k} \subseteq\{1, \ldots, n\}$ for all $0<j_{1}<\cdots<j_{k} \leq n$. We will occasionally abuse the notation, and we will use $e_{\emptyset}=e_{0}=1$ for the unit of the algebra $\mathrm{Cl}_{n}$, but it is important not to confuse the unit of $\mathrm{Cl}_{n}, e_{0}=1$, with the unit of the field $\mathbb{R}$. With this construction it is clear that $\operatorname{dim}\left(\mathrm{Cl}_{n}\right)=2^{n}$. We follow the (non standard) selection of Ahlfors [1], by identifying the vectors of $\mathbb{R}^{n}$ with the elements spanned by $e_{0}, \ldots, e_{n-1}$. There are three important involutions in $\mathrm{Cl}_{n}$ similar to the complex conjugation. The main involution defined as $a \rightarrow a^{\prime}$ which replaces each $e_{i}$ by $-e_{i}$ (so that $e_{J}^{\prime}=(-1)^{|J|} e_{J}$ ) the reversion $a \rightarrow a^{*}$, which reverses the order of each multi-index in $e_{J}$, and their combination, the Clifford conjugation, $a \rightarrow \bar{a}:=a^{*}=a^{* \prime}$. An important subgroup of the Clifford algebra is $\Gamma_{n}$, the Clifford group, which is the set of invertible elements in $\mathrm{Cl}_{n}$ that can be represented as products of non-zero vectors in $\mathbb{R}^{n}$.

With our particular identification of vectors $\mathbb{R}^{n}$ in $\mathrm{Cl}_{n}$, the Clifford product $x y$ between two vectors $x, y \in \mathbb{R}^{n}, x=\sum_{i=0}^{n-1} x_{i} e_{i}, y=\sum_{i=0}^{n-1} y_{i} e_{i}$, 
can be written as

$$
\begin{aligned}
x y= & \left(x_{0} y_{0}-\sum_{i=1}^{n-1} x_{i} y_{i}\right) e_{0}+\sum_{i=1}^{n-1}\left(x_{0} y_{i}+x_{i} y_{0}\right) e_{i} \\
& +\sum_{i=1}^{n-1}\left(x_{i} y_{j}-x_{j} y_{i}\right) e_{i j} .
\end{aligned}
$$

Remark 3.1. (Geometric Algebra) An algebraic structure closely related to the Clifford algebra is the exterior algebra (or Grassmann algebra), $\Lambda(V)$, generated by the elements $e_{1}, \ldots, e_{n}$ with the wedge product (or exterior product) defined by the relations $e_{i} \wedge e_{i}=0$ and $e_{i} \wedge e_{j}=-e_{j} \wedge e_{i}, i \neq j$. Basic building blocks are the exterior products of $k$-vectors $\left\{v_{i}\right\}_{i=1}^{k}$, also referred to as $k$-blades $v_{1} \wedge \cdots \wedge v_{k}$, and linear combinations of blades, called multivectors. A useful property of the exterior product is the efficient algebraic representation of basic geometrical entities. More precisely, if we have a $k$ dimensional homogeneous subspace $W$ spanned by $k$ vectors $\left\{w_{i}\right\}_{i=1}^{k}$, the $k$-blade $w=w_{1} \wedge \cdots \wedge w_{k}$ can be used to represent $W$ as

$$
x \in W \Longleftrightarrow x \wedge w=0 \text {. }
$$

For instance, if $x$ is an element of the line spanned by $v \in V$, we have $x=\lambda v$, and this property can be related to the equality $x \wedge v=0$. Additionally, a vector $x$ lies in a plane spanned by $v$ and $u$ iff $x \wedge v \wedge u=0$. But this framework is not only restricted to homogeneous subspaces: further generalizations can be considered with the same algebraic efficiency for more elaborate geometrical objects [3]. Particularly important tools in this field are efficient algorithms (the join and meet operations) for constructing the intersection and union of subspaces.

\subsection{Möbius Transforms in $\mathbb{R}^{n}$}

Möbius transformations, and the general concept of conformal maps, have appeared in a wide range of theoretical and practical applications, ranging from airfoil design in aerodynamics to modern problems in brain surface conformal mapping. In the context of this work, we are interested in their flexible geometrical properties for designing invertible nonlinear maps with computationally efficient algebraic characteristics. Recall that a Möbius transformation is a function $\mathfrak{f}: \hat{\mathbb{C}} \rightarrow \hat{\mathbb{C}}$, with $\hat{\mathbb{C}}=\mathbb{C} \cup\{\infty\}$, of the form

$$
\mathfrak{f}(z):=\frac{a z+b}{c z+d},
$$

where $\left(\begin{array}{ll}a & b \\ c & d\end{array}\right) \in \operatorname{Mat}(2, \mathbb{C})$, with $a d-b c \neq 0$. During the last century, Möbius transforms were generalized by Vahlen, Maass, and Ahlfors to arbitrary vector spaces using Clifford algebras and Clifford groups [1]:

Definition 3.1. For the vector space $V=\mathbb{R}^{n}$, a Möbius transform $\mathfrak{f}: \hat{\mathbb{R}}^{n} \rightarrow$ $\hat{\mathbb{R}}^{n}$, with $\hat{\mathbb{R}}^{n}:=\mathbb{R}^{n} \cup\{\infty\}$, is defined as $\mathfrak{f}(v)=(a v+b) /(c v+d)$, where the Clifford matrix $H_{\mathfrak{f}}:=\left(\begin{array}{ll}a & b \\ c & d\end{array}\right) \in \operatorname{Mat}\left(2, C l_{n}\right)$ is required to satisfy the following three conditions. 
1) $a, b, c, d \in \Gamma_{n} \cup\{0\}$,

2) $a b^{*}, c d^{*} \in \mathbb{R}^{n}$,

3) $\Delta(\mathfrak{f}):=a d^{*}-b c^{*} \in \mathbb{R}^{*}$.

Remark 3.2. (The Vahlen-Maass Theorem) The Vahlen-Maass Theorem states that the set of Clifford matrices, denoted by $\mathrm{SL}_{2}\left(\Gamma_{n}\right)$, forms a group under the matrix multiplication. Moreover, the product $H_{\mathfrak{f}} H_{\mathfrak{g}}$ corresponds to composition of Möbius transforms $\mathfrak{f} \circ \mathfrak{g}$. The Vahlen-Maass Theorem also relates Clifford matrices with the concept of Möbius maps as composition of similarities and inversions over the unit sphere). The expression $\Delta(\mathfrak{f})$ is sometimes denominated pseudo determinant.

\subsection{An Explicit Construction}

In this section, we provide a simple and explicit construction of a Möbius transform in $\mathbb{R}^{n}$, satisfying the three conditions in Definition 3.1. This yields an algorithm for designing Möbius transformations matching our specific needs, as for instance, the construction of hyperbolic transformations from two given fixed points.

Remark 3.3. (Constructing Möbius transforms in $\hat{\mathbb{C}}$ ) For designing a Möbius transform in $\hat{\mathbb{C}}$ such that $\mathfrak{f}(x)=u, \mathfrak{f}(y)=v, \mathfrak{f}(z)=w$ we can use the following standard construction which consist of first mapping the points $x, y, z$ to 0,1 and $\infty$ using $\mathfrak{f}_{1}(x)=0, \mathfrak{f}_{1}(y)=1$, and $\mathfrak{f}_{1}(z)=\infty$, with $($ for $\mathbf{z} \in \mathbb{C}$ )

$$
\mathfrak{f}_{1}(\mathbf{z}):=\frac{(\mathbf{z}-x)(y-z)}{(\mathbf{z}-z)(y-x)}, H_{\mathfrak{f}_{1}}:=\left(\begin{array}{cc}
y-z & x(z-y) \\
y-x & z(x-y)
\end{array}\right) .
$$

If we consider also a second map $\mathfrak{f}_{2}$ with $\mathfrak{f}_{2}(u)=0, \mathfrak{f}_{2}(v)=1, \mathfrak{f}_{2}(w)=\infty$, we can now construct $\mathfrak{f}:=\mathfrak{f}_{2}^{-1} \circ \mathfrak{f}_{1}$ with $\mathfrak{f}(x)=u, \mathfrak{f}(y)=v, \mathfrak{f}(z)=w$, using $H_{\mathfrak{f}}:=H_{\mathfrak{f}_{2}}^{-1} H_{\mathfrak{f}_{1}}$.

The general idea of this construction can be extended to $\mathbb{R}^{n}$, but some constraints need to be considered. The following is a particular strategy that can be used in our framework.

Lemma 3.1. Given a vector $x \in \mathbb{R}^{n}, n>1$, we can construct a Möbius transform $\mathfrak{f}$ such that $\mathfrak{f}(x)=0, \mathfrak{f}(y)=1$ and $\mathfrak{f}(z)=\infty$, for $y, z \in \mathbb{R}^{n}$ provided that the following three conditions are satisfied.

1) $z_{i}=k x_{i}, i=1, \ldots, n-1$, for some $k \in \mathbb{R}^{*}$,

2) $y=\alpha x+\beta z$, for some $\alpha, \beta \in \mathbb{R}, \quad \alpha+\beta=1$,

3) $3\left(x_{0}-z_{0}\right)^{2}=\sum_{i=1}^{n-1}\left(x_{i}-z_{i}\right)^{2}$.

Proof: Using the ideas of Remark 3.3, we analyze the three conditions in Definition 3.1. Recall that with our particular identification of $\mathbb{R}^{n}$ in $\mathrm{Cl}_{n}$ we have $x=\sum_{i=0}^{n-1} x_{i} e_{i}, y=\sum_{i=0}^{n-1} y_{i} e_{i}$, and $z=\sum_{i=0}^{n-1} z_{i} e_{i}$. For an triple $x, y, z$ fulfilling the conditions 1 ) and 2 ), the coefficients

$$
\begin{array}{ll}
a=y-z, & b=x(z-y), \\
c=y-x, & d=z(x-y),
\end{array}
$$


fulfill the first requirement in Definition 3.1. As for the second condition in Definition 3.1, we have

$$
\begin{aligned}
& a b^{*}=(y-z)(z-y)^{*} x^{*}=-(y-z)^{2} x, \\
& c d^{*}=(y-x)(x-y)^{*} z^{*}=-(y-x)^{2} z,
\end{aligned}
$$

and the pseudo determinant is given by

$$
\Delta(\mathfrak{f})=a d^{*}-b c^{*}=(y-z)(x-y)(z-x) .
$$

Now, $(y-z)^{2} x \in \mathbb{R}^{n}$ and $(y-x)^{2} z \in \mathbb{R}^{n}$, if

$$
\begin{aligned}
& (\mathbf{y}-\mathbf{z})^{2} \wedge \mathbf{x}=0, \\
& (\mathbf{y}-\mathbf{x})^{2} \wedge \mathbf{z}=0,
\end{aligned}
$$

where $\mathbf{x}$ is the non-real part of $x$, i.e., $\mathbf{x}=\sum_{i=1}^{n-1} x_{i} e_{i}$. These two conditions can be fulfilled, if we require (as described in Remark 3.1) the vector $\mathbf{y}-\mathbf{z}$ to be an element of the line spanned by $\mathbf{x}$, and the vector $\mathbf{y}-\mathbf{x}$ to be an element spanned by $\mathbf{z}$. Therefore we have $\mathbf{z}=k \mathbf{x}, k \in \mathbb{R}$. If we select a vector $y=\alpha x+\beta z, \alpha+\beta=1$, we have

$$
a d^{*}-b c^{*}=(y-z)(x-y)(z-x)=\alpha \beta(z-x)^{3} .
$$

Now, for a vector $v=\sum_{i=0}^{n-1} v_{i} e_{i} \in \mathbb{R}^{n}$ we have

$$
v^{3}=\left(v_{0}^{3}-3 v_{0} \sum_{i=1}^{n-1} v_{i}^{2}\right) e_{0}+\sum_{j=1}^{n-1}\left(3 v_{0}^{2}-\sum_{i=1}^{n-1} v_{i}^{2}\right) v_{j} e_{j} .
$$

Therefore, $v^{3} \in \mathbb{R}$ iff $3 v_{0}^{2}=\sum_{i=1}^{n-1} v_{i}^{2}$, which implies that $(z-x)^{3} \in \mathbb{R}$ if and only if $3\left(x_{0}-z_{0}\right)^{2}=\sum_{i=1}^{n-1}\left(x_{i}-z_{i}\right)^{2}$. We finally notice that by combining these conditions we also need $\mathbf{z}=k \mathbf{x}$, for $k \in \mathbb{R}^{*}$.

Note that the we can relax our above conditions on $y-z$ (resp. $y-x$ ). But the statement on Lemma 3.1 is sufficient for our next objective. In particular, we can now construct a variety of useful linear or nonlinear maps in $\mathbb{R}^{n}$ as hyperbolic Möbius transforms based on the next proposition.

Proposition 3.1. For a pair $u, v \in \mathbb{R}^{n}, n>1$, and two vectors $w_{i}=\alpha_{i} u+\beta_{i} v$, $i=1,2$, with $\alpha_{i}+\beta_{i}=1, \alpha_{i}, \beta_{i} \in \mathbb{R}$, there exists a Möbius transform $\mathfrak{f}: \hat{\mathbb{R}}^{n} \rightarrow \hat{\mathbb{R}}^{n}$ satisfying $\mathfrak{f}(u)=u, \mathfrak{f}(v)=v$, and $\mathfrak{f}\left(w_{1}\right)=w_{2}$.

Proof: This follows as a straightforward consequence from Lemma 3.1 and the Vahlen-Maass theorem which relates the group structure of $\mathrm{SL}_{2}\left(\Gamma_{n}\right)$ with the composition of Möbius transforms in $\mathbb{R}^{n}$ (see Remark 3.2). More precisely, following along the lines of Remark 3.3, we first consider the translation $\mathfrak{t}$, with $\mathfrak{t}((u+v) / 2)=0$, followed by a rotation $\mathfrak{r}$, such that the third condition of Lemma 3.1 is fullfilled. Now we can use Lemma 3.1 for constructing two Möbius maps $\mathfrak{f}_{1}, \mathfrak{f}_{2}$ with $\mathfrak{f}_{1}(\mathfrak{r}(\mathfrak{t}(u)))=0, \mathfrak{f}_{1}\left(\mathfrak{r}\left(\mathfrak{t}\left(w_{1}\right)\right)\right)=1, \mathfrak{f}_{1}(\mathfrak{r}(\mathfrak{t}(v)))=\infty$, and $\mathfrak{f}_{2}(\mathfrak{r}(\mathfrak{t}(u)))=0, \mathfrak{f}_{2}\left(\mathfrak{r}\left(\mathfrak{t}\left(w_{2}\right)\right)\right)=1, \mathfrak{f}_{2}(\mathfrak{r}(\mathfrak{t}(v)))=\infty$. Using now the VahlenMaass Theorem the composition $\mathfrak{f}:=\mathfrak{t}^{-1} \mathfrak{r}^{-1} \mathfrak{f}_{2}^{-1} \mathfrak{f}_{1} \mathfrak{r t}$ is a Möbius transform and its Clifford matrix is given by $H_{\mathfrak{f}}=H_{\mathfrak{t}}^{-1} H_{\mathfrak{r}}^{-1} H_{\mathfrak{f}_{2}}^{-1} H_{\mathfrak{f}_{1}} H_{\mathfrak{r}} H_{\mathfrak{t}}$. 


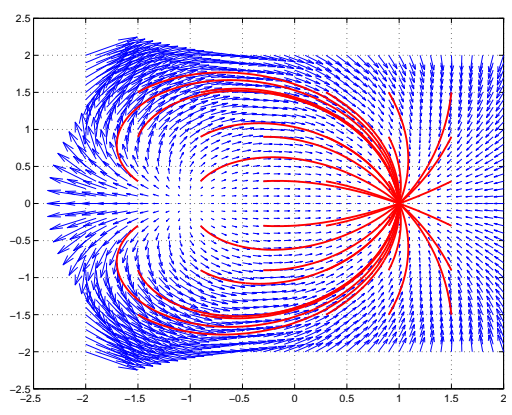

Figure 1. Hyperbolic Möbius transform with two fixed points (one attractive and one repulsive).

By Proposition 3.1, we can now construct hyperbolic Möbius transforms by calibrating its vector field (see Fig. 1 ) with the vectors $w_{1}$ and $w_{2}$. These kind of maps have useful properties for shrinking or separating clusters. In general, with Lemma 3.1 we can also design other maps, as rotations in $\mathbb{R}^{n}$, with alternative constructions to more classical strategies such as the well known Procrustes problem.

\section{Computational Experiment}

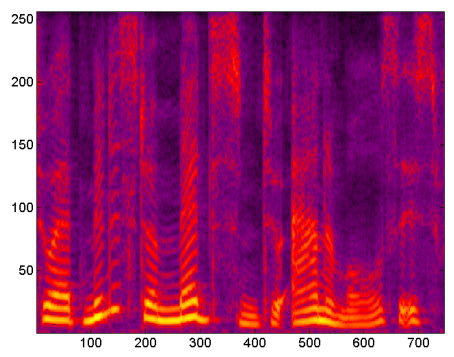

(a)

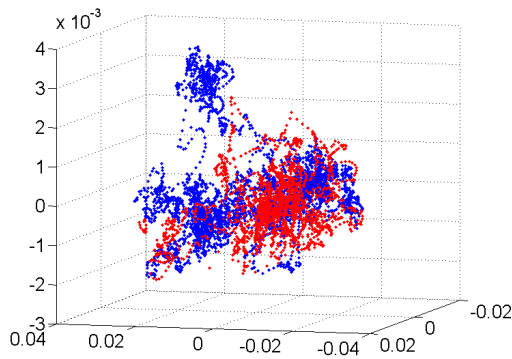

(b)

Figure 2. (a) Spectrogram of speech signal (b) PCA projection with consonants (red) vs vocals (blue).

As a basic application of our tools, we present the problem of improving the classification and detection of consonants in a speech signal. We use a speech signal $f=g+h$, where $g$ corresponds to the harmonic components (vocals), and $h$ corresponds to the non-harmonic components (consonants). We construct the spectrogram $T\left(X_{f}\right)=\left\{T\left(x_{i}\right)\right\}_{i}$ of $f$, depicted in Fig. 2a, where $T$ is the power spectrum (using the Fourier transform) applied to each 


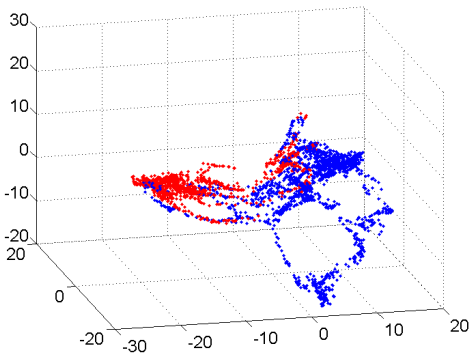

(a)

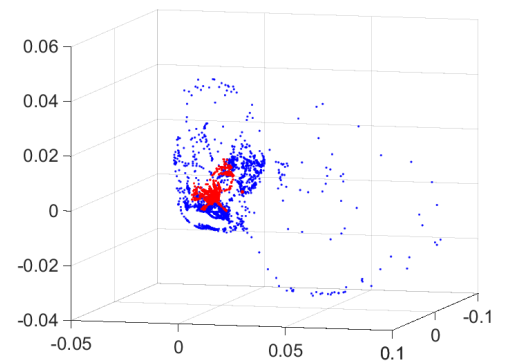

(b)

FiguRE 3. (a) Isomap projection and consonants (red) vs vocal (blue) components (b) Möbius transformation of (a).

segment $x_{i}$. The vectors $x_{i}$ are constructed as a sequence of small consecutive chunks of the signal $f$ (as performed in short term Fourier analysis). We use dimensionality reduction methods (principal component analysis (PCA) and isomap), represented as maps $R: \mathbb{R}^{n} \rightarrow \mathbb{R}^{d}$. The resulting projections in $\mathbb{R}^{d}, d=3$, are depicted in Fig. 2b, and Fig. 3a, where the consonant and vocal elements are depicted with red and blue clusters $\left(R\left(T\left(X_{g}\right)\right)\right.$ and $\left.R\left(T\left(X_{h}\right)\right)\right)$.

We can see that isomap in Fig. 3a provides a better cluster separation in comparison to PCA as depicted in Fig. 2b. Indeed, the corresponding consonant cluster (in red) is not as spread as in the case of the PCA projection of Fig. 2b. However, our design of a Möbius map with an attractive fixed point in the center of the consonant cluster further improves the geometric separation between the two clusters: in Fig. 3b one can observe how the consonant patch in red is shrinked to a smaller region, providing a better scenario for classification routines.

In order to provide a quantitative evaluation of these results, we estimate the success rate achieved when using a classification routine with these different datasets (Isomap point cloud $R_{i}\left(T\left(X_{f}\right)\right.$ ) vs Isomap-Möbius point cloud $R_{m}\left(T\left(X_{f}\right)\right)$, see Fig. 3 . We use a standard support vector machine (svm) algorithm for differentiating the consonants from the vocal components of the signal. In Fig.4 we display a plot with the corresponding success rate when using svm with a polynomial kernel. The $x$-axis of the plot represents the amount of samples from the datasets $R_{i}\left(T\left(X_{f}\right), R_{m}\left(T\left(X_{f}\right)\right.\right.$ used for training the svm algorithm. The $y$-axis represents the corresponding success rate when svm attempts to estimate if a point belongs either to the consonant or vocal cluster. The dotted curve of Fig.4 indicates the success rate of svm with a polynomical kernel when using the Isomap dataset $R_{i}\left(T\left(X_{f}\right)\right.$ ), see Fig. 3a. The solid curve of Fig.4 represents the success rate of svm with a polynomial kernel for the Möbius-Isomap dataset $R_{m}\left(T\left(X_{f}\right)\right)$, see Fig. 3b. As can be seen in the plot of Fig.4, the Möbius-Isomap dataset $R_{m}\left(T\left(X_{f}\right)\right)$ provides a better success rate (higher than $88 \%$ ) when differentiating between 
consonant and vocal components. The Isomap dataset $R_{i}\left(T\left(X_{f}\right)\right)$ achieves a success rate of approximately $82 \%$ in average. Obtaining these particular results displayed in Fig.3, and the corresponding svm classification evaluation Fig.4, requires a careful tuning when selecting the Möbius transform for the algorithm. Future work should investigate a more adequate automatic selection of parameters for the Möbius transformation. Here, we only indicate the possibilities and potentials of our machinery. The corresponding code for these numerical experiments is available in the homepage of the first author http: //www.math.tu-berlin.de/?110812.

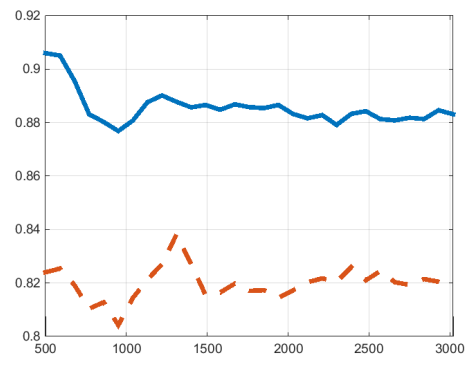

FigurE 4. SVM succcess rate of Möbius-Isomap vs Isomap projections

\section{Acknowledgments}

We are grateful to the anonymous reviewers who provided important remarks and suggestions.

\section{References}

[1] L.V. Ahlfors. Möbius transformations in $\mathbb{R}^{n}$ expressed through $2 \times 2$ matrices of Clifford numbers. Complex Variables and Elliptic Equations, 5(2):215-224, 1986.

[2] G. Carlsson. Topology and data. American Mathematical Society, 46(2):255$308,2009$.

[3] L. Dorst, D. Fontijne, and S. Mann. Geometric algebra for computer science: an object-oriented approach to geometry. Morgan Kaufmann, 2009.

[4] H.B. Lawson and M.L. Michelson. Spin Geometry. Princeton University Press, Princeton, New Jersey, 1989.

[5] J.A. Lee and M. Verleysen. Nonlinear dimensionality reduction. Springer Verlag, 2007.

[6] P. Niyogi, S. Smale, and S. Weinberger. Finding the homology of submanifolds with high confidence from random samples. Discrete and Computational Geometry, 39(1):419-441, 2008.

[7] A. Zomorodian and G. Carlsson. Computing persistent homology. Discrete Comput. Geom., 33(2):249-274, 2005. 
Mijail Guillemard*1

TU Berlin, Department of Mathematics, 10623 Berlin, Germany

e-mail: guillemard@math.tu-berlin.de

Armin Iske

University of Hamburg, Department of Mathematics, 20146 Hamburg, Germany e-mail: iske@math.uni-hamburg.de

Udo Zölzer

Helmut-Schmidt-University, Dept of Signal Processing, 22043 Hamburg, Germany e-mail: udo.zoelzer@hsu-hamburg.de

\footnotetext{
${ }^{1 *}$ Corresponding author
} 